\title{
Characterization of VOCs and their related atmospheric processes in a central Chinese city during severe ozone pollution periods
}

\section{Bowei Li et al.}

Correspondence to: Steven Sai Hang Ho (stevenho@hkpsrl.org) and Sunling Gong (gongsl@cma.gov.cn)

The copyright of individual parts of the supplement might differ from the CC BY 4.0 License. 


\section{Quality control and quality assurance $(\mathrm{QC} / \mathrm{QA})$}

In order to alleviate contamination from previous samples, canisters had to be repeatedly cleaned using humidified zero air before sampling. In order to inspect the cleanliness and vacuum in canisters, the pre-cleaned canisters, after storing for $24 \mathrm{~h}$, were analyzed using GC-MS according to the same analytical procedures used to analyze the field samples. The canisters without any contamination were used for sampling. No obvious disturbances due to improperly activities (such as smoking, spray fumes, etc.) of sample collectors were present during the sampling events. Daily calibration of the GC-MSD/FID was performed using 2 ppbv standard mixtures to ensure the consistency and sensitivity of the system. The deviation in standard values was within $\pm 20 \%$. Abnormal QC/QA data with extremely high or low responses was recalibrated until the deviation was within the acceptable range.

\section{PMF model}

In brief, PMF is a diagnostic method involving multivariate analysis, and involves decomposing the integrated sample data (VOCs in this study) into two matrices, namely the source profiles and the source contributions (Jaars et al., 2018). The method takes advantage of other observation data, such as wind direction and speed. Detailed information on the application of PMF can be referred to the publications cited above and to the PMF 5.0 user manual (U.S. EPA, 2014).

Based on the chemical mass balance between the input concentrations of VOCs and the chemical profiles, PMF regarded the ambient data $x_{\mathrm{ij}}$, namely the concentration of $j^{\text {th }}$ constituent in $i^{\text {th }}$ sample, as the gross values contributed by $p$ sources according to Eq. (1).

$$
X_{\mathrm{i} j}=\sum_{k=1}^{p} g_{i k} f_{k j}+e_{i j}
$$

where $g_{\mathrm{ik}}$ stands for the contribution of $k^{\text {th }}$ factor in the $i^{\text {th }}$ sample, while $f_{\mathrm{kj}}$ is the load of $j^{\text {th }}$ compound in the $k^{\text {th }}$ source and the $e_{\mathrm{ij}}$ is the relevant residual.

In order to avoid negative source contributions, a penalty function was adopted for constraints. Each data point can be individually weighed in the model, while the 
samples with lots of missing values were excluded.

Based on the algorithm for uncertainties (U), expressed as $Q$ values, the stability of running results was assessed according to Eq. (2).

$$
Q=\sum_{i=1}^{n} \sum_{j=1}^{m}\left[\frac{x_{i j}-\sum_{k=1}^{p} g_{i k} f_{k j}}{u_{i j}}\right]^{2}
$$

where $u_{\mathrm{ij}}$ stands for the uncertainty of the $j^{\text {th }}$ compound in $i^{\text {th }}$ sample.

In the PMF model, uncertainty is a function to evaluate the deviations in sampling and analysis procedure (Paatero, 2007). The uncertainty (U) can be calculated based on Eq. (3) (Polissar et al., 1998).

$$
\mathrm{U}= \begin{cases}\sqrt{(E F \times \text { concentration })^{2}+(M D L)^{2}} & (\text { conc. }>\mathrm{MDL}) \\ \frac{5}{6} M D L & (\text { conc } \leq \mathrm{MDL})\end{cases}
$$

where $E F$ signifies the error fraction that equals 100 times of the percentage uncertainty.

\section{Source identification}

Source profiles showed that the sites had similar regional characteristic. There was a strong common source (factor 1 ) for $\mathrm{C}_{2}-\mathrm{C}_{5} \mathrm{n}$-alkanes and certain amounts of $\mathrm{C}_{2}$ - $\mathrm{C}_{4}$ alkenes, benzene, toluene, acetylene and $\mathrm{NO}_{2}$. It is reported that $\mathrm{i}$-pentane and aromatics (such as, benzene, toluene, ethylbenzene and m/p-xylene) are usually originated from gasoline evaporation (Watson et al., 2001), while isobutane and n-butane are emitted from vehicles fueled with LPG/CNG (Li et al., 2017;Liu et al., 2008), in addition, the ratio of toluene close to 2 is defined as vehicle emission.

The second factor was characterized based upon significant loading of toluene, ethylbenzene, m/p-xylene, o-xylene and m-ethyltoluene. According to previous studies (Yuan et al., 2010;Wang et al., 2014), toluene and $\mathrm{C}_{8}$ - C9 aromatics were the major VOCs emitted from paint applications. Therefore, factor 2 was assigned as the solvent use. This is consistent with the real situation that there were widespread road paving and building constructions in Zhengzhou. In addition, car decoration, printing, and 
56 furniture manufacturing, which are associated with the use of adhesives, were also

57 included in this source category.

Factor 3 was dominated by c/t-2-butene, 1-butene and c/t-2-pentene. According to our unpublished data, these species were abundant in ambient air next to oil gas stations. This factor was thus categorized as the oil evaporation. The profile at MEM was contained more $\mathrm{C}_{8}-\mathrm{C}_{10}$ alkanes and m-ethyltoluene, which were abundant in diesel vapor, indicating the heavier diesel evaporation at this site.

The fourth factor shows a dominant loading of cyclohexane, styrene, 2-methylhexane, 3-methylhexane and 2,3-dimethylpentane. The first three VOCs were identified as the components heavily impacted by petrochemical industries (Jobson et al., 2004). Therefore, this source was defined as the petrochemical. propane and several amounts of $\mathrm{C}_{7}-\mathrm{C}_{10}$ alkanes. Both coal and biomass burning could produce large amounts of acetylene (Ho et al., 2009), benzene, ethylene and propylene. According to lower level of toluene in this factor, this factor was referred to as the coal+biomass burning.

Factor 6 was distinguished by extremely high compositions of isoprene, a species mainly produced by vegetation through photosynthesis (Millet et al., 2016). Even though it can be emitted from traffic-related sources (Yuan et al., 2009), this can be possibly ignored by its poor correlations with other source makers of vehicle exhaust (e.g., i-pentane and ethylene). Therefore, this factor was identified as the biogenic emissions. 
Table S1. Detailed information of monitoring equipment for $\mathrm{SO}_{2}, \mathrm{CO}, \mathrm{NO}_{\mathrm{x}}, \mathrm{O}_{3}$ and meteorological

80 factors

\begin{tabular}{|c|c|c|}
\hline Targets & Equipment & Model \\
\hline $\mathrm{SO}_{2}$ & Pulsed Fluorescence $\mathrm{SO}_{2}$ Analyzer & Model 43i, Thermo, Inc. \\
\hline $\mathrm{CO}$ & Gas Filter Correlation CO Analyzer & Model 48i, Thermo, Inc. \\
\hline $\mathrm{NO}-\mathrm{NO}_{2}-\mathrm{NOx}$ & Chemiluminescence NO-NO $2-\mathrm{NOx}$ Analyzer & Model 42i, Thermo, Inc. \\
\hline $\mathrm{O}_{3}$ & ultra-violet (UV) photometric $\mathrm{O}_{3}$ analyzer & Model 49i, Thermo, Inc. \\
\hline Meteorological data & multi-parameter automatic weather station & Milos 520, Vaisala, Inc. \\
\hline
\end{tabular}

81 


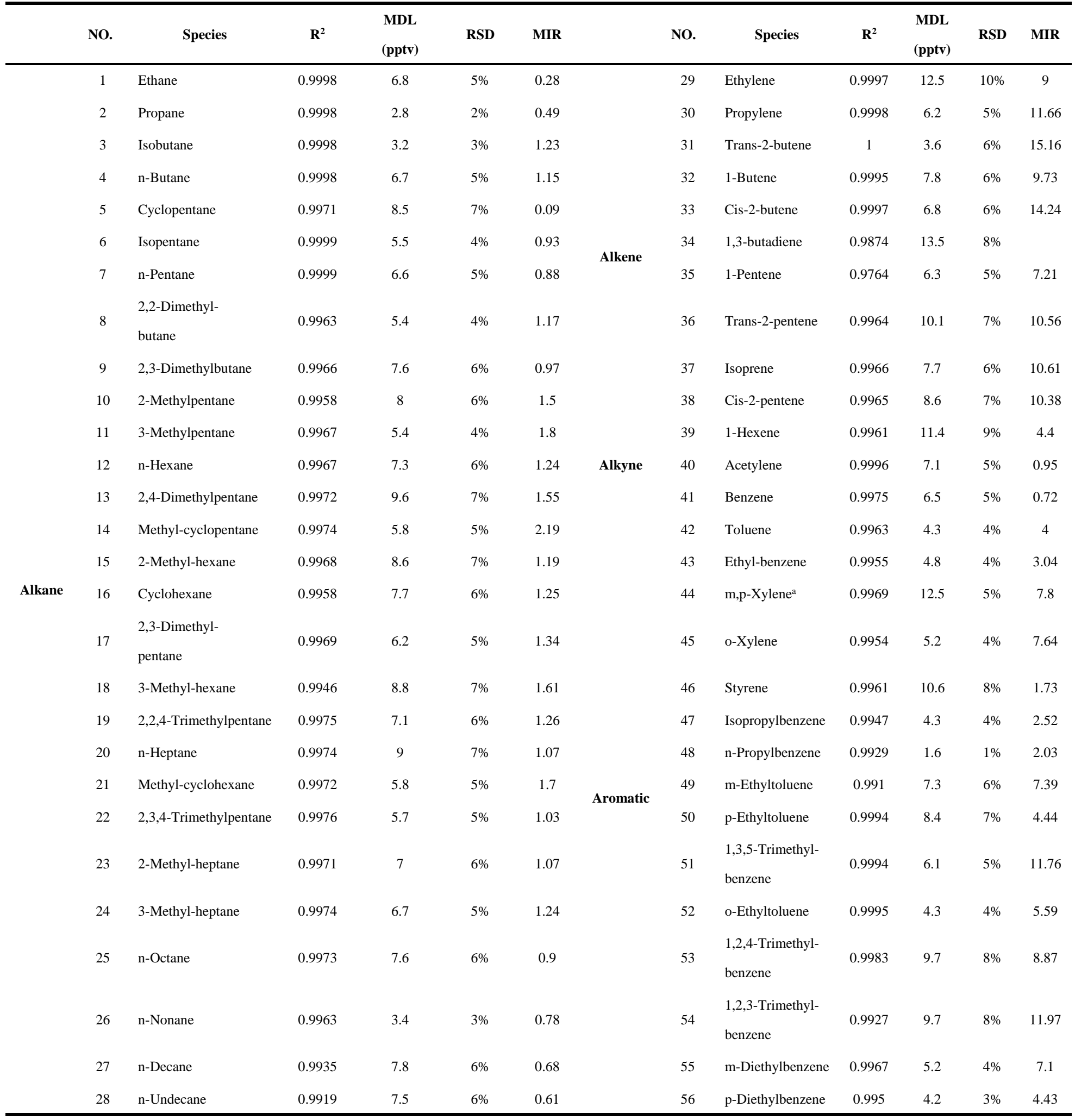

$84 \quad{ }^{\mathrm{a}} \mathrm{m}$-Xylene and $p$-Xylene are co-eluted in the chromatographic separation. 
87 Table S3. The correlation coefficient $\left(\mathrm{R}^{2}\right)$ between observed and predicted values for each compound at the four sites

\begin{tabular}{|c|c|c|c|c|}
\hline species & JK & MEM & YH & GS \\
\hline ethane & 0.73 & 0.59 & 0.87 & 0.68 \\
\hline propane & 0.82 & 0.89 & 0.87 & 0.45 \\
\hline isobutane & 0.97 & 0.87 & 0.64 & 0.93 \\
\hline n-butane & 0.73 & 0.93 & 0.93 & 0.66 \\
\hline isopentane & 0.66 & 0.40 & 0.81 & 0.76 \\
\hline n-pentane & 0.84 & 0.65 & 0.63 & 0.86 \\
\hline methyl-cyclopentane & 0.98 & 0.86 & 0.77 & 0.89 \\
\hline 2-methyl-hexane & 0.99 & 0.97 & 0.85 & 0.98 \\
\hline cyclohexane & 1.00 & 0.95 & 0.92 & 0.95 \\
\hline 2,3-dimethyl-pentane & 0.99 & 0.99 & 0.97 & 0.99 \\
\hline 3-methyl-hexane & 0.99 & 0.97 & 0.88 & 0.99 \\
\hline 2,2,4-trimethyl-pentane & 0.77 & 0.89 & 0.81 & 0.80 \\
\hline n-heptane & 0.99 & 0.87 & 0.96 & 0.77 \\
\hline n-octane & 0.77 & 0.88 & 0.61 & 0.72 \\
\hline n-decane & 0.52 & 0.73 & 0.15 & 0.70 \\
\hline ethylene & 0.83 & 0.92 & 0.33 & 0.91 \\
\hline propylene & 0.85 & 0.88 & 0.81 & 0.87 \\
\hline trans-2-butene & 0.90 & 0.87 & 0.93 & 0.73 \\
\hline 1-butene & 0.93 & 0.95 & 0.94 & 0.74 \\
\hline cis-2-butene & 0.93 & 0.96 & 0.97 & 0.80 \\
\hline trans-2-pentene & 0.87 & 0.93 & 0.97 & 0.84 \\
\hline isoprene & 0.98 & 0.90 & 0.90 & 0.88 \\
\hline cis-2-pentene & 0.87 & 0.96 & 0.94 & 0.74 \\
\hline acetylene & 0.43 & 0.80 & 0.73 & 0.82 \\
\hline benzene & 0.96 & 0.79 & 0.39 & 0.91 \\
\hline toluene & 0.94 & 0.85 & 0.86 & 0.52 \\
\hline ethyl-benzene & 0.91 & 0.93 & 0.99 & 0.97 \\
\hline m,p-xylene ${ }^{\mathrm{a}}$ & 0.88 & 0.99 & 0.97 & 0.88 \\
\hline o-xylene & 0.94 & 0.99 & 0.97 & 0.88 \\
\hline styrene & 0.74 & 0.82 & 0.66 & 0.87 \\
\hline m-ethyltoluene & 0.74 & 0.93 & 0.94 & 0.81 \\
\hline $\mathrm{NO}_{2}$ & 0.81 & 0.57 & 0.73 & 0.65 \\
\hline
\end{tabular}

$89 \quad{ }^{a} m$-Xylene and $p$-Xylene are co-eluted in the chromatographic separation.

90

91

92 
Table S4 Error estimation summary results, i.e. BS mapping for the four sites

(a) JK

\begin{tabular}{cccccccc}
\hline & biogenic & $\begin{array}{c}\text { oil gas } \\
\text { evaporation }\end{array}$ & petrochemical & $\begin{array}{c}\text { vehicle } \\
\text { emission }\end{array}$ & $\begin{array}{c}\text { solvent } \\
\text { usage }\end{array}$ & $\begin{array}{c}\text { coal+biomass } \\
\text { burning }\end{array}$ & Unmapped \\
\hline Boot Factor 1 & 100 & 0 & 0 & 0 & 0 & 0 & 0 \\
Boot Factor 2 & 0 & 100 & 0 & 0 & 0 & 0 & 0 \\
Boot Factor 3 & 0 & 1 & 98 & 1 & 0 & 0 & 0 \\
Boot Factor 4 & 0 & 0 & 0 & 100 & 0 & 0 & 0 \\
Boot Factor 5 & 0 & 0 & 0 & 0 & 100 & 0 & 0 \\
Boot Factor 6 & 0 & 0 & 0 & 3 & 0 & 97 & 0
\end{tabular}

96

(b) MEM

\begin{tabular}{cccccccc}
\hline & $\begin{array}{c}\text { vehicle } \\
\text { emission }\end{array}$ & biogenic & petrochemical & $\begin{array}{c}\text { solvent } \\
\text { usage }\end{array}$ & $\begin{array}{c}\text { coal+biomass } \\
\text { burning }\end{array}$ & $\begin{array}{c}\text { oil gas } \\
\text { evaporation }\end{array}$ & Unmapped \\
\hline Boot Factor 1 & 100 & 0 & 0 & 0 & 0 & 0 & 0 \\
Boot Factor 2 & 0 & 100 & 0 & 0 & 0 & 0 & 0 \\
Boot Factor 3 & 1 & 0 & 99 & 0 & 0 & 0 & 0 \\
Boot Factor 4 & 0 & 0 & 0 & 100 & 0 & 0 & 0 \\
Boot Factor 5 & 0 & 0 & 0 & 0 & 100 & 0 & 0 \\
Boot Factor 6 & 0 & 0 & 0 & 0 & 0 & 100 & 0 \\
\hline
\end{tabular}

97

\begin{tabular}{lccccccc} 
(c) YH & & & & & & \\
& solvent & petrochemical & $\begin{array}{c}\text { vehicle } \\
\text { emission }\end{array}$ & $\begin{array}{c}\text { coal+biomass } \\
\text { burning }\end{array}$ & $\begin{array}{c}\text { oil gas } \\
\text { evaporation }\end{array}$ & biogenic & Unmapped \\
\hline Boot Factor 1 & 99 & 0 & 1 & 0 & 0 & 0 & 0 \\
Boot Factor 2 & 1 & 99 & 0 & 0 & 0 & 0 & 0 \\
Boot Factor 3 & 0 & 2 & 94 & 2 & 2 & 0 & 0 \\
Boot Factor 4 & 0 & 0 & 0 & 99 & 1 & 0 & 0 \\
Boot Factor 5 & 0 & 0 & 0 & 1 & 99 & 0 & 0 \\
Boot Factor 6 & 0 & 0 & 0 & 0 & 0 & 100 & 0 \\
\hline
\end{tabular}

98

(d) GS

\begin{tabular}{lccccccc}
\hline & petrochemical & biogenic & $\begin{array}{c}\text { oil gas } \\
\text { evaporation }\end{array}$ & $\begin{array}{c}\text { coal+biomass } \\
\text { burning }\end{array}$ & $\begin{array}{c}\text { vehicle } \\
\text { emission }\end{array}$ & $\begin{array}{c}\text { solvent } \\
\text { usage }\end{array}$ & Unmapped \\
\hline Boot Factor 1 & 94 & 0 & 0 & 1 & 0 & 2 & 3 \\
Boot Factor 2 & 0 & 100 & 0 & 0 & 0 & 0 & 0 \\
Boot Factor 3 & 1 & 0 & 91 & 1 & 1 & 4 & 2 \\
Boot Factor 4 & 0 & 0 & 0 & 100 & 0 & 0 & 0 \\
Boot Factor 5 & 0 & 0 & 4 & 0 & 82 & 10 & 4 \\
Boot Factor 6 & 0 & 0 & 0 & 0 & 0 & 100 & 0 \\
\hline
\end{tabular}


105

106

107

108

109

110

111

112

113

Table S5. The ratio of Q/Q(exp) at factor size ranged from 3-9 at the four sites

\begin{tabular}{ccccc}
\hline & JK & MEM & YH & GS \\
\hline 3 & 2.62 & 2.12 & 2.43 & 2.73 \\
4 & 2.07 & 1.62 & 1.96 & 2.19 \\
5 & 1.56 & 1.23 & 1.57 & 1.86 \\
6 & 1.27 & 1.01 & 1.23 & 1.69 \\
7 & 1.15 & 0.80 & 1.10 & 1.40 \\
8 & 1.03 & 0.71 & 1.01 & 1.24 \\
9 & 0.94 & 0.66 & 0.92 & 1.18 \\
\hline
\end{tabular}

Table S6. The average mixing ratios of $\mathrm{SO}_{2}, \mathrm{NO}_{\mathrm{x}}, \mathrm{CO}$ and $\mathrm{O}_{3}$ from May to September 2017

\begin{tabular}{lcccc}
\hline & $\mathbf{S O}_{2}(\mathbf{p p b})$ & $\mathbf{N O x}(\mathbf{p p b})$ & $\mathbf{C O}(\mathbf{p p m})$ & $\mathbf{O}_{\mathbf{3}}(\mathbf{p p b})$ \\
\hline May & 9.55 & 35.76 & 0.70 & 86.73 \\
June & 5.49 & 31.76 & 0.71 & 99.32 \\
July & 2.52 & 20.62 & 0.69 & 76.27 \\
August & 2.98 & 25.48 & 0.89 & 76.92 \\
September & 5.72 & 52.60 & 0.96 & 61.31 \\
\hline
\end{tabular}

Table S7. Identified source categories by PMF and their corresponding markers

\begin{tabular}{cccc}
\hline Source & Source category & Markers & Reference \\
\hline 1 & vehicle emission & C2-C5 alkanes, $\mathrm{NO}_{2}$ & (Watson et al., 2001) \\
2 & $\begin{array}{c}\text { coal+biomass } \\
\text { burning }\end{array}$ & ethane, ethylene, acetylene, benzene & 2013) \\
& (Liu et al., 2008;Zhang et al., \\
3 & solvent usage & toluene, ethylbenzene, xylenes & (Yuan et al., 2010;Wang et al., \\
4 & oil gas evaporation & C4-C5 alkenes, n-heptane & 2014) \\
5 & petrochemical & methyl-cyclopentane, cyclohexane, 3-Methyl-hexane, & (Wiu et al., 2008; Jobson et al., \\
6 & biogenic & 2-Methyl-hexane, styrene & 2004) \\
\end{tabular}



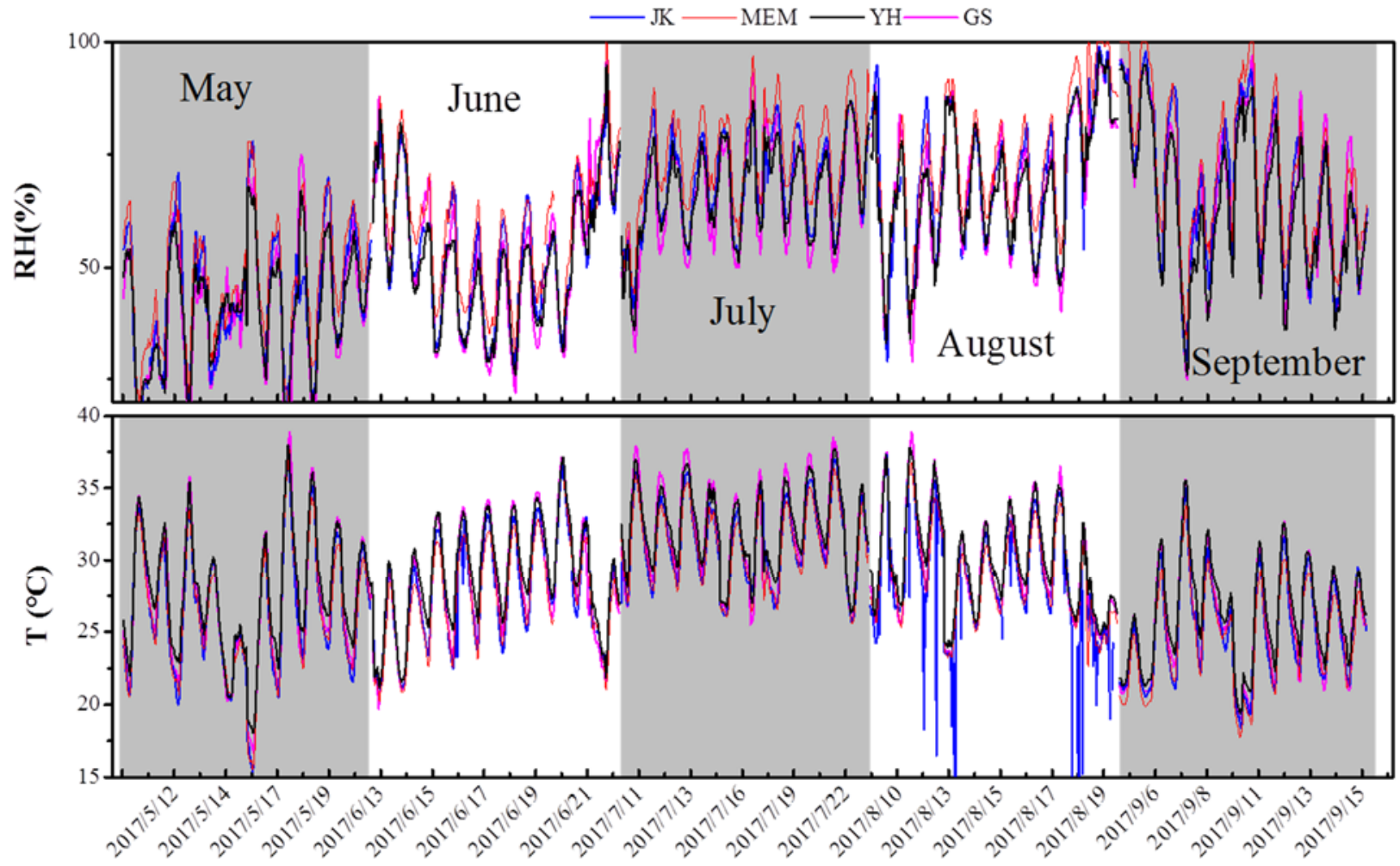

Fig. S1. The temperature and relative humidity at each site during sampling period 
JK

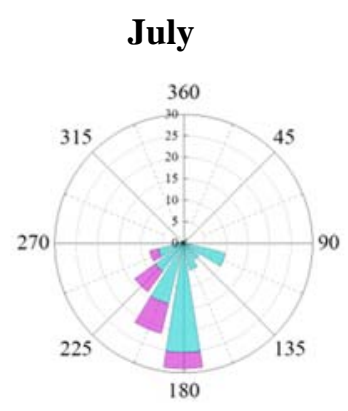

MEM

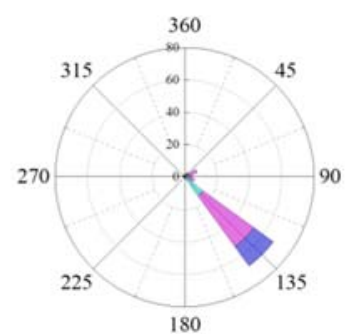

YH

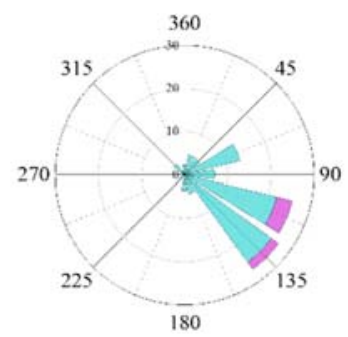

GS

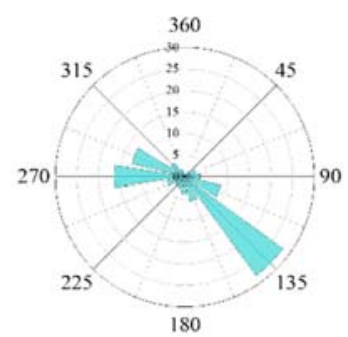

August
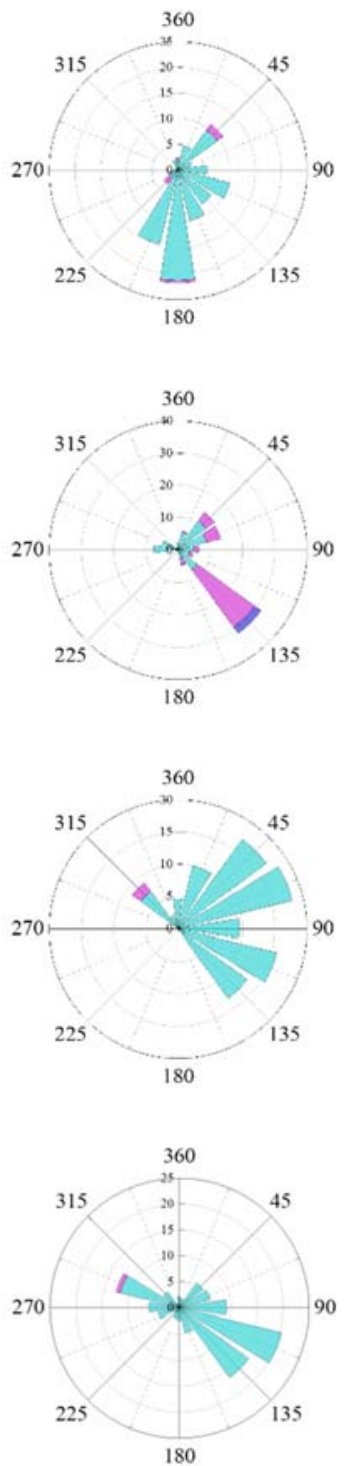

September
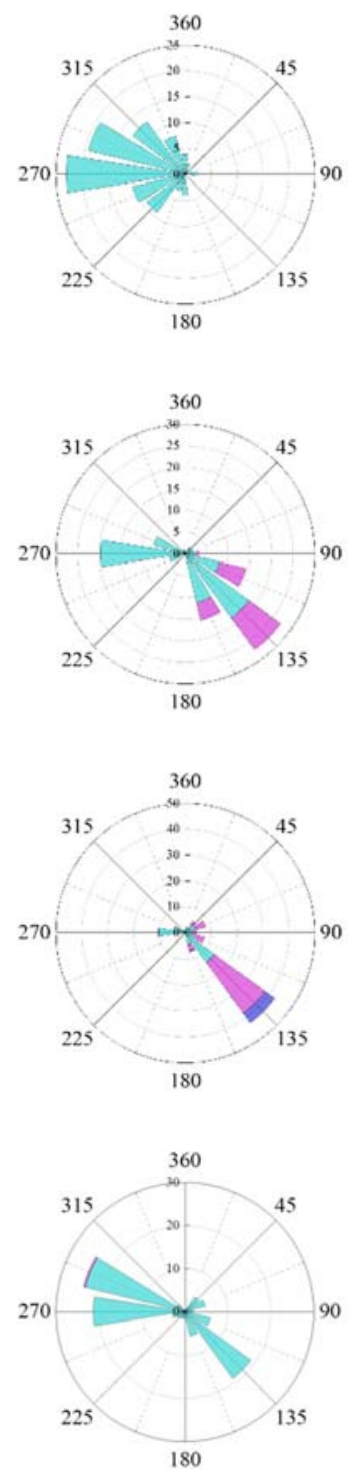

Fig. S2. The wind distribution at each site in July, August and September 


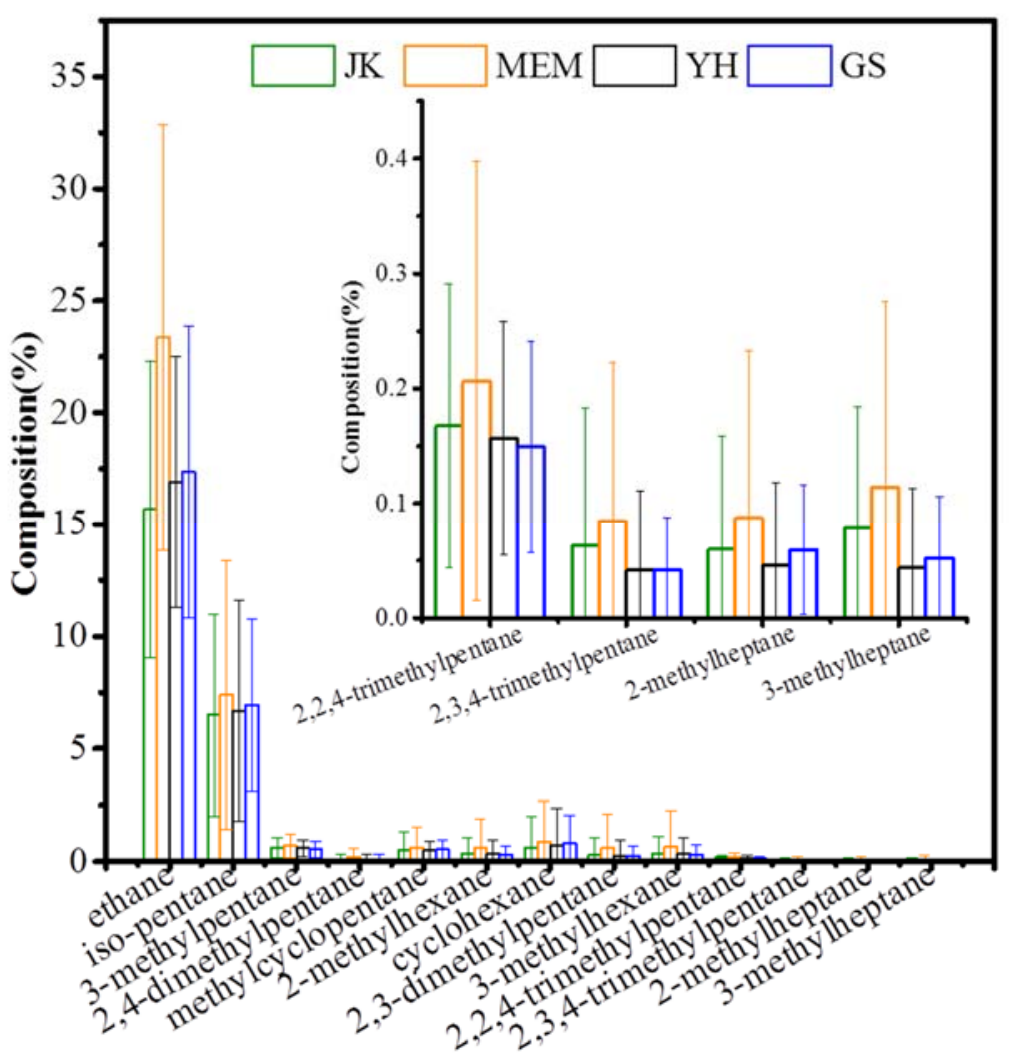



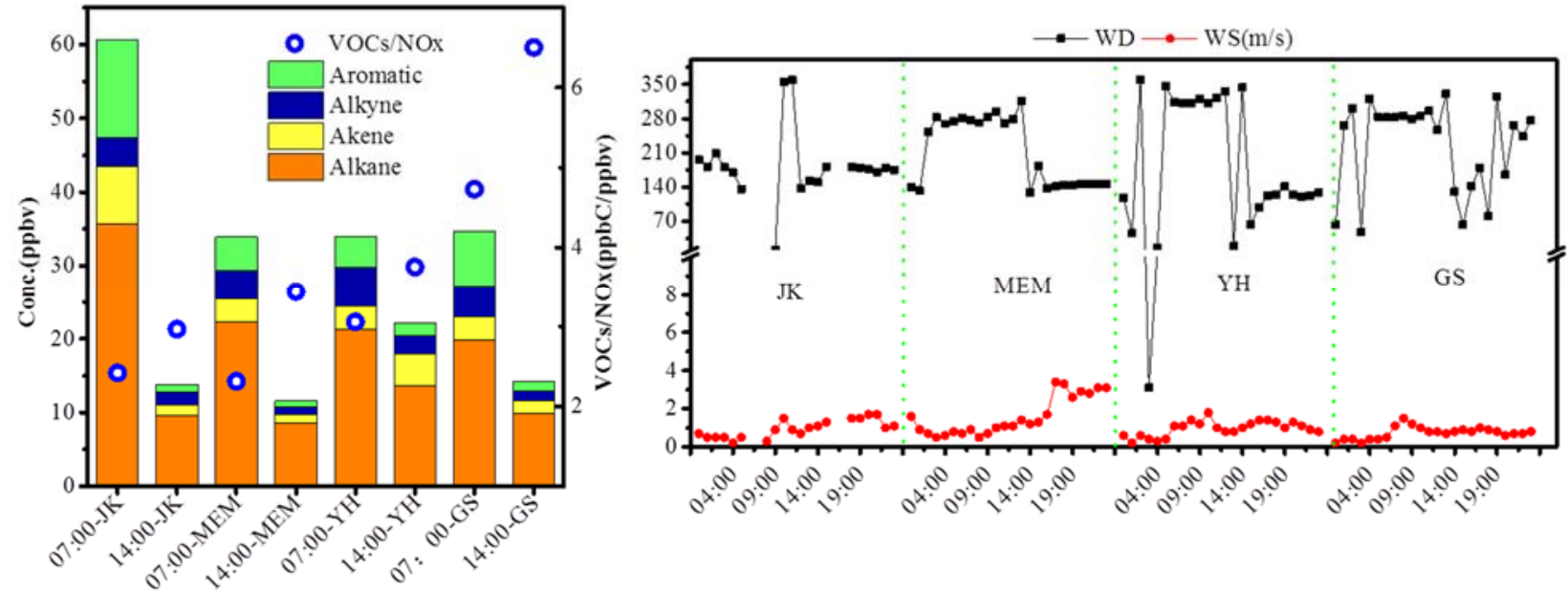

Fig.S4. Temporal variation of compositions, $\mathrm{VOCs} / \mathrm{NO}_{\mathrm{x}}$, wind direction and wind speed on $10^{\text {th }}$ of 

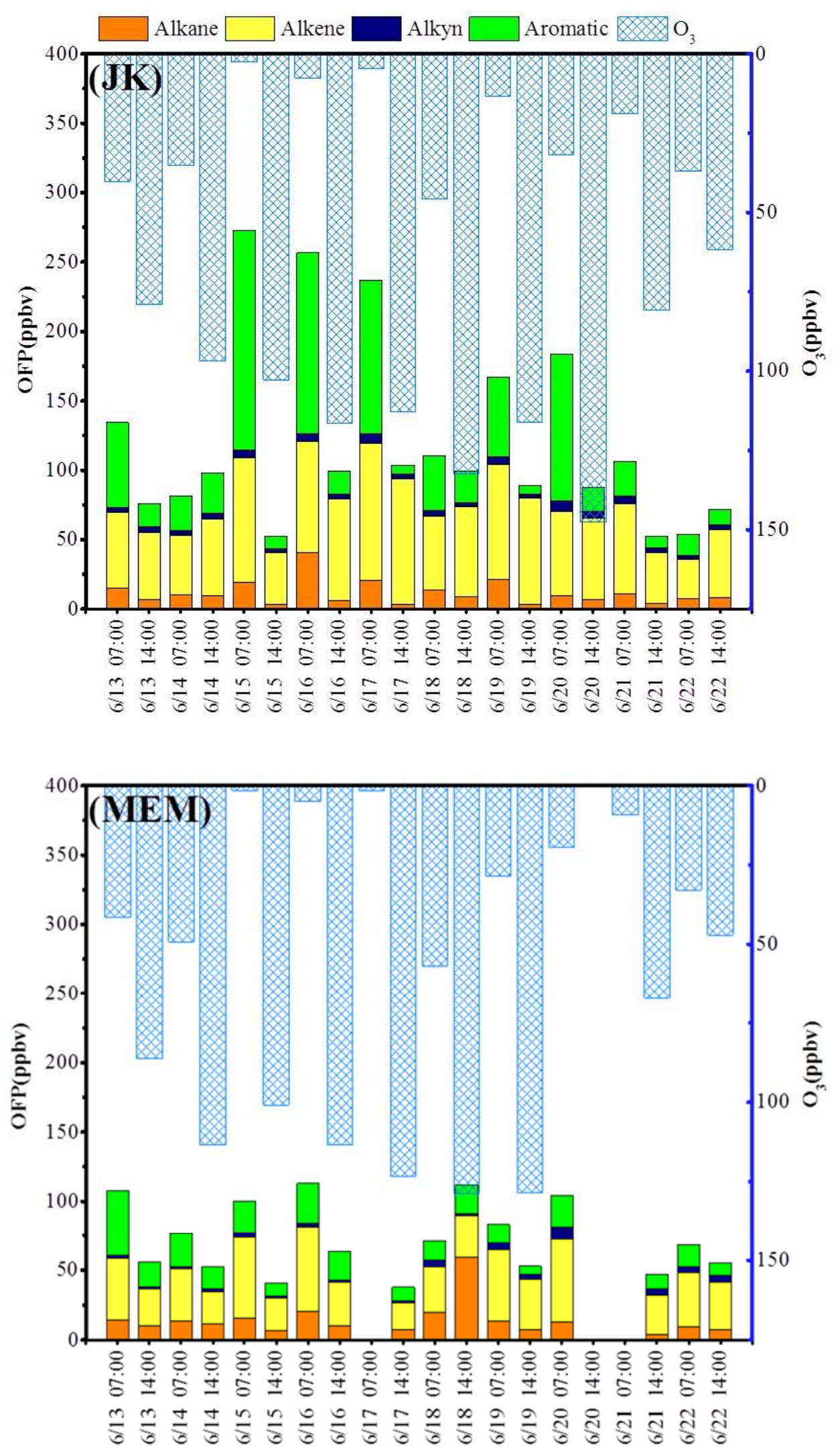

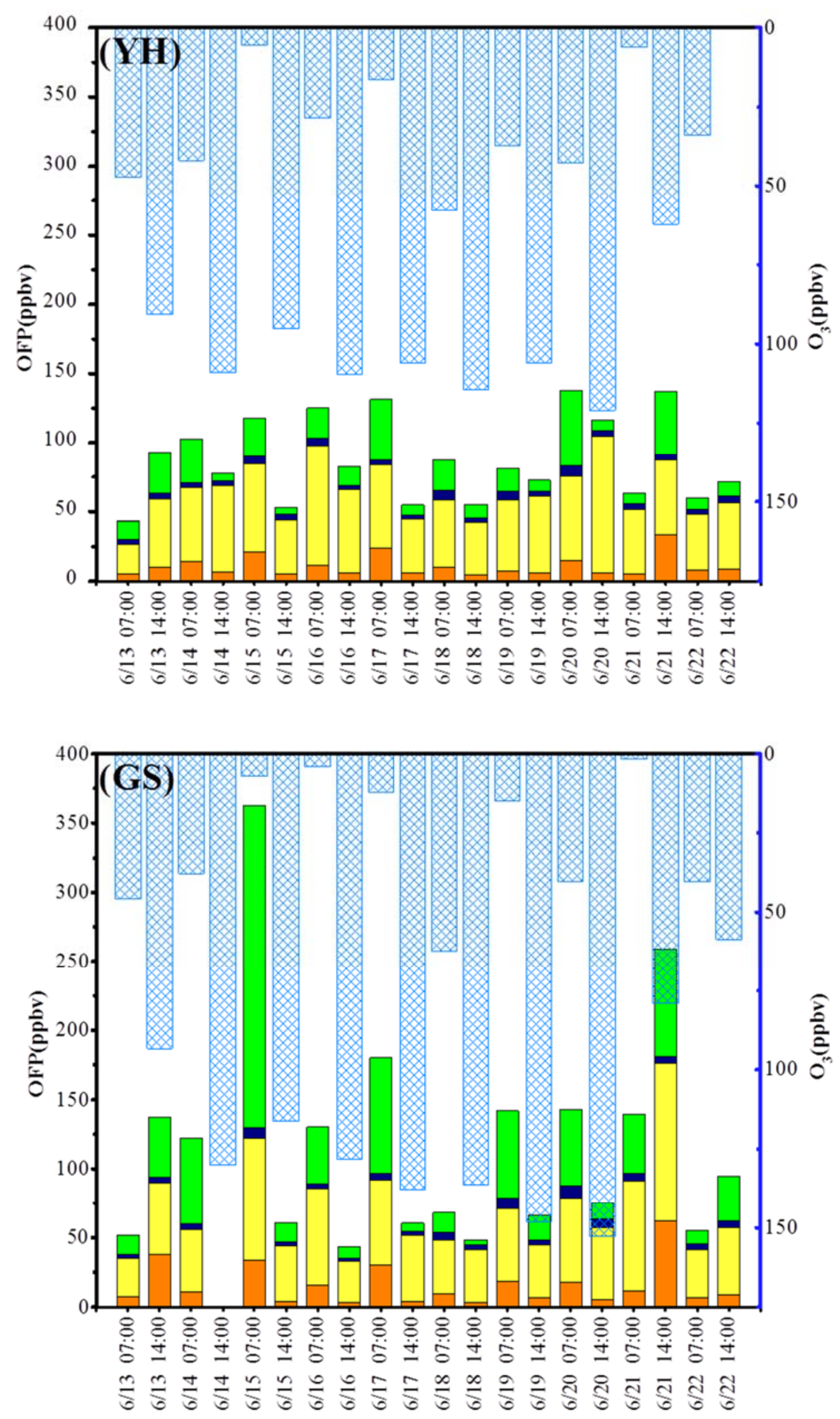


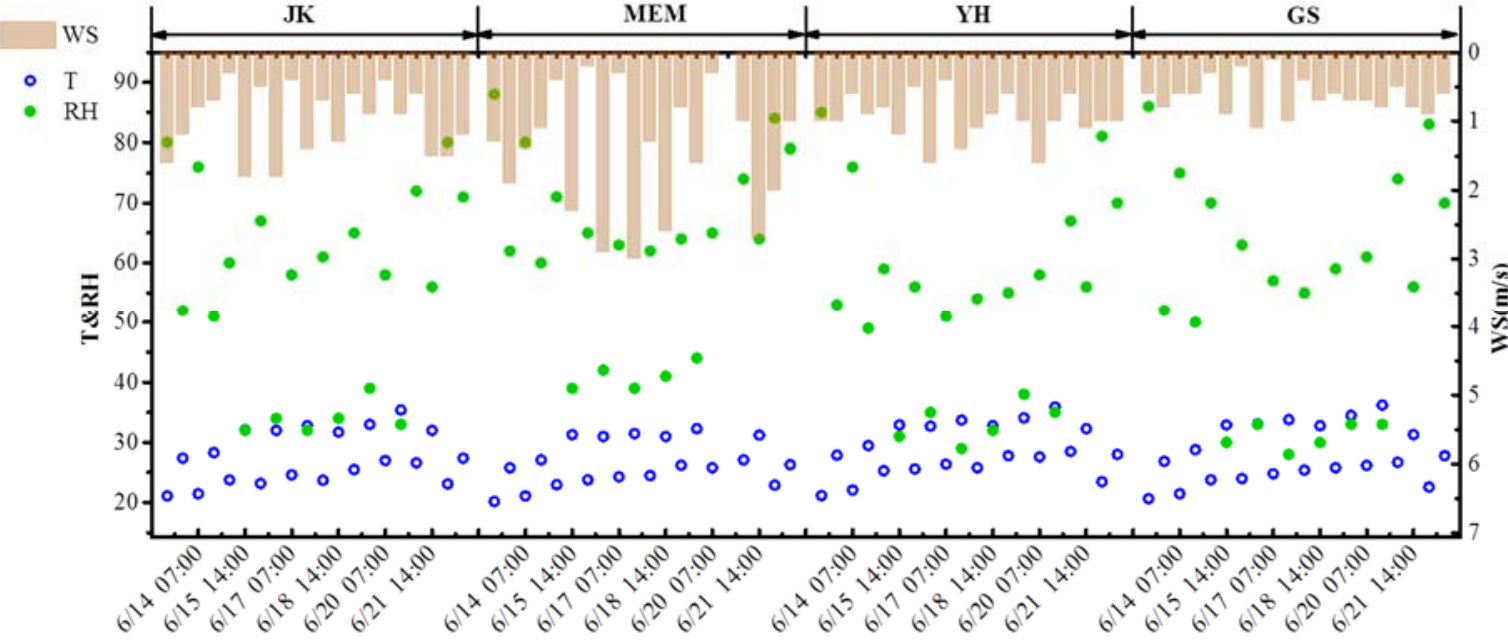

Fig.S6. Spatio-temporal variations in meterological factors in June 


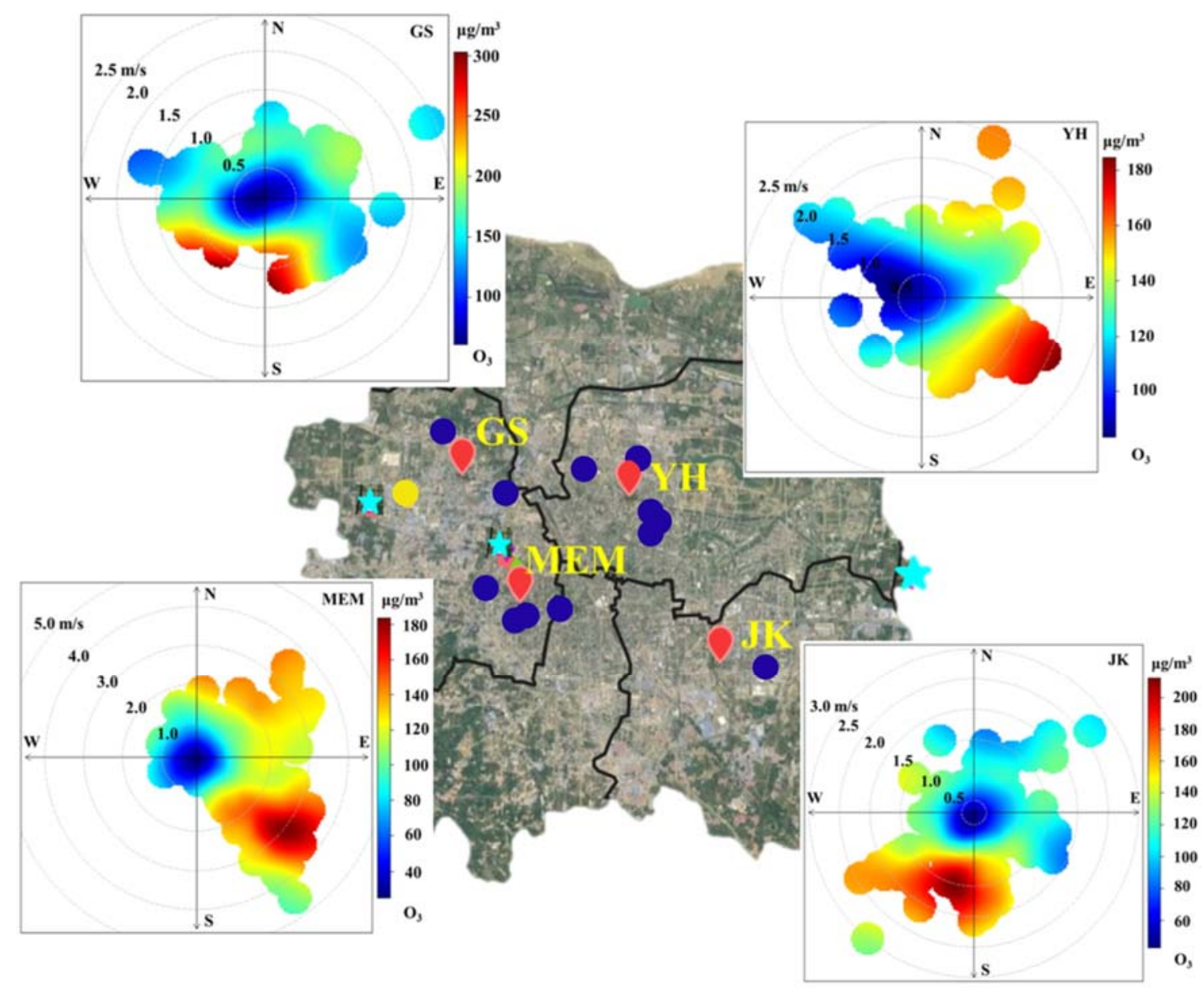

Fig. S7. Relationship among $\mathrm{O}_{3}\left(\mu \mathrm{g} \mathrm{m}^{-3}\right)$, wind direction and wind speed $\left(\mathrm{m} \mathrm{s}^{-1}\right)$ during sampling period in June, 2017 


\section{Reference}

Ho, K. F., Lee, S. C., Ho, W. K., Blake, D. R., Cheng, Y., Li, Y. S., Ho, S. S. H., Fung, K., Louie, P. K. K., and Park, D.: Vehicular emission of volatile organic compounds (VOCs) from a tunnel study in Hong Kong, Atmos. Chem. Phys., 9, 7491-7504, 2009.

Jaars, K., Vestenius, M., van Zyl, P. G., Beukes, J. P., Hellén, H., Vakkari, V., Venter, M., Josipovic, M., and Hakola, H.: Receptor modelling and risk assessment of volatile organic compounds measured at a regional background site in South Africa, Atmos. Environ., 172, 133-148, https://doi.org/10.1016/j.atmosenv.2017.10.047, 2018.

Jobson, B. T., Berkowitz, C. M., Kuster, W. C., Goldan, P. D., Williams, E. J., Fesenfeld, F. C., Apel, E. C., Karl, T., Lonneman, W. A., and Riemer, D.: Hydrocarbon source signatures in Houston, Texas: Influence of the petrochemical industry, J. Geophys. Res., 109, D24305, https://doi.org/10.1029/2004jd004887, 2004.

Li, B., Ho, S. S. H., Xue, Y., Huang, Y., Wang, L., Cheng, Y., Dai, W., Zhong, H., Cao, J., and Lee, S.: Characterizations of volatile organic compounds (VOCs) from vehicular emissions at roadside environment: The first comprehensive study in Northwestern China, Atmos. Environ., 161, 1-12, https://doi.org/10.1016/j.atmosenv.2017.04.029, 2017.

Liu, Y., Shao, M., Fu, L., Lu, S., Zeng, L., and Tang, D.: Source profiles of volatile organic compounds (VOCs) measured in China: Part I, Atmos. Environ., 42, 6247-6260, https://doi.org/10.1016/j.atmosenv.2008.01.070, 2008.

Millet, D. B., Baasandorj, M., Hu, L., Mitroo, D., Turner, J., and Williams, B. J.: Nighttime Chemistry and Morning Isoprene Can Drive Urban Ozone Downwind of a Major Deciduous Forest, Environ. Sci. Technol., 50, 4335-4342, https://doi.org/10.1021/acs.est.5b06367, 2016.

Paatero, P.: User's Guide for positive Matrix Factorization programs PMF2 and PMF3, part 1-2: Tutorial, 19 -21. University of Helsinki, Helsinki, Finland, 2007.

Polissar, A. V., Hopke, P. K., Paatero, P., Malm, W. C., and Sisler, J. F.: Atmospheric aerosol over Alaska: 2. Elemental composition and sources, J. Geophys. Res.-Atmos., 103, 19045-19057, https://doi.org/10.1029/98jd01212, 1998.

US EPA: EPA Positive Matrix Factorization (PMF) 5.0 Fundamentals and User Guide, http://www.epa.gov/heasd/documents/ EPA. PMF.5.0.User.Guide. pdf, 2014.

Wang, H.-l., Jing, S.-a., Lou, S.-r., Hu, Q.-y., Li, L., Tao, S.-k., Huang, C., Qiao, L.-p., and Chen, C.-h.: Volatile organic compounds (VOCs) source profiles of on-road vehicle emissions in China, Sci. Total Environ., 607-608, 253-261, https://doi.org/10.1016/j.scitotenv.2017.07.001, 2017.

Wang, H., Qiao, Y., Chen, C., Lu, J., Qiao, L., and Lou, S.: Source Profiles and Chemical Reactivity of Volatile Organic Compounds from Solvent Use in Shanghai, China, Aerosol Air Qual. Res., 14, 301-310, https://doi.org/10.4209/aaqr.2013.03.0064, 2014.

Watson, J. G., Chow, J. C., and Fujita, E. M.: Review of volatile organic compound source apportionment by chemical mass balance, Atmos. Environ., 35, 1567-1584, 2001.

Yuan, B., Shao, M., Lu, S., and Wang, B.: Source profiles of volatile organic compounds associated with solvent use in Beijing, China, Atmos. Environ., 44, 1919-1926, https://doi.org/10.1016/j.atmosenv.2010.02.014, 2010.

Yuan, Z., Lau, A. K. H., Shao, M., Louie, P. K. K., Liu, S. C., and Zhu, T.: Source analysis of volatile organic compounds by positive matrix factorization in urban and rural environments in Beijing, J. Geophys. Res., 114, D00G15, https://doi.org/10.1029/2008jd011190, 2009. 
Zhang, Y., Shao, M., Lin, Y., Luan, S., Mao, N., Chen, W., and Wang, M.: Emission inventory of carbonaceous pollutants from biomass burning in the Pearl River Delta Region, China, Atmos. Environ., 76, 189-199, https://doi.org/10.1016/j.atmosenv.2012.05.055, 2013.

191 